\title{
EFEITO DO EXERCÍCIO FÍSICO SOBRE A DISLIPIDEMIA
}

\author{
EFFECT OF PHYSICAL EXERCISE ON DYSLIPIDEMIA
}

\section{BIANCA TOSTES SOARES A $^{*}$ LUILA VIANNA RODRIGUES AMARAL B $^{*}$, DIOGO CORREIA CARDOZO ${ }^{*}$, EMERSON RODRIGUES DUARTE ${ }^{D^{*}}$}

biancatts@hotmail.comª, luillavianna@yahoo.com.br ${ }^{\mathrm{b}}$, dcardozoef@gmail.com ${ }^{\mathrm{c}}$, emerson01duarte@gmail.com ${ }^{\mathrm{d}}$ Faculdade Metodista Granbery, Juiz de Fora, $\mathrm{MG}^{*}$

\section{RESUMO}

Introdução: A dislipidemia é um fator de risco para o desenvolvimento de aterosclerose e doenças vasculares. Em contra partida, o exercício físico é considerado uma terapia contra a dislipidemia. Objetivo: Analisar os efeitos de um programa de exercícios físicos no perfil lipídico de indivíduos dislipidêmicos de ambos os sexos participantes de um programa multidisciplinar de reabilitação cardiopulmonar e metabólica (RCPM). Métodos: Foi feito uma análise retrospectiva de um banco de dados de pacientes atendidos na RCPM. O treinamento físico realizado consistia de uma combinação entre exercícios de força e aeróbicos realizados duas vezes por semana durante 10 meses. Resultados: Após o período de intervenção foi observado melhora em todos os parâmetros metabólicos investigados $(\mathrm{P} \leq 0,05)$. Conclusão: $\mathrm{A}$ adesão em um programa de RCPM para portadores de dislipidemia, por aproximadamente 10 meses, praticado duas vezes na semana, mostrou melhora no perfil de colesterol total, HDL, LDL e triglicérides.

Palavras-chave: Exercício físico; dislipidemia; saúde vascular

\begin{abstract}
Introduction: Dyslipidemia is a risk factor for the development of atherosclerosis and vascular diseases. In contrast, physical exercise is considered a therapy against dyslipidemia. Objective: To analyze the effects of a physical exercise program on the lipid profile of dyslipidemic individuals of both sexes participating in a multidisciplinary cardiopulmonary and metabolic rehabilitation program (RCPM). Methods: A retrospective analysis of a database of patients seen in the RCPM was performed. The physical training performed consisted of a combination of strength and aerobic exercises performed twice a week for 10 months. Results: After the intervention period an improvement in all the metabolic parameters investigated was observed $(\mathrm{P} \leq 0,05)$. Conclusion: Adherence in a RCPM program for patients with dyslipidemia for approximately 10 months, performed twice a week, showed an improvement in the total cholesterol, HDL, LDL and triglyceride profiles.
\end{abstract}

Keywords: Physical Exercise. Dyslipidemia. Vascular Health. 


\section{INTRODUÇÃO}

De acordo a Organização Pan-Americana de Saúde (OPAS) a incidência de doenças cardiovasculares vem aumentando de forma abrupta ao longo dos últimos anos, sendo considerada como o principal fator de mortalidade mundial ${ }^{1}$. Dados da Sociedade Brasileira de Cardiologia ${ }^{2}$ (SBC) demonstram estimativas no Brasil em torno de 17,5 milhões de óbitos computados por doenças cardiovasculares no ano de 2012, sendo que destes, 7,4 milhões dos óbitos foram ocasionados por doenças coronarianas e 6,7 milhões devido a acidentes vasculares cerebrais ${ }^{1,2}$.

É sabido que o comportamento sedentário é um fator ambiental responsável pelo aumento destas complicações, pois, quando associado aos maus hábitos alimentares podem contribuir para o desenvolvimento de doenças vasculares, tais como, doença arterial coronariana (DAC) e aterosclerose que são originadas pelo acúmulo de placas de ateroma no interior das artérias influenciando negativamente na saúde vascular ${ }^{2}$. Nesse contexto, a dislipidemia que é definida como um distúrbio no metabolismo dos lipídios (colesterol total, triglicerídeos) e das lipoproteínas (lipoproteínas de alta densidade - HDL, lipoproteínas de baixa densidade - LDL) pode cooperar para a formação e/ou evolução deste quadro devido seu efeito potencial de agressão ao endotélio e consequente favorecimento da aterosclerose e doenças mais severas $^{2,3}$. Em contra partida, a prática de exercícios físicos é considerado como uma das principais intervenções não farmacológicas contra a dislipidemia e doenças vasculares, pois exerce influência na melhora do perfil lipídico, condutância vascular, complacência arterial, modulação autonômica com melhora do balanço simpatovagal, diminuição dos níveis de pressão arterial, redução dos níveis de gordura corporal, normalização da glicemia, entre outras respostas benéficas ${ }^{4-8}$.

Com base na V Diretriz Brasileira sobre dislipidemias e prevenção da aterosclerose é recomendado que exercícios aeróbicos como caminhadas, corridas leves, ciclismo e natação devam ser realizados de três a seis vezes na semana, em sessões com duração de 30 a 60 minutos com intensidade entre $60 \%$ e $80 \%$ da frequência cardíaca máxima, bem como a realização de exercícios resistidos em complemento aos exercícios aeróbicos com intensidade correspondente a $50 \%$ da força de contração voluntária máxima². Entretanto, mesmo existindo estas recomendações sobre a duração das sessões de treinamento, intensidade e tipo de exercício, as informações existentes ainda são incipientes para a prescrição com o objetivo da melhora do perfil lipídico. Por esta razão, foi objetivo do presente estudo analisar os efeitos de um programa de exercícios físicos no perfil lipídico de indivíduos dislipidêmicos de ambos os sexos participantes de um programa multidisciplinar de reabilitação cardiopulmonar e metabólica.

\section{METODOLOGIA}

\section{ASPECTOS ÉTICOS}

O projeto foi aprovado por um Comitê de Ética em Pesquisa com seres humanos (CAAE ${ }^{\circ}$ 67165417.3.0000.5089). Obteve-se autorização da empresa privada de saúde complementar para utilização de dados oriundos de prontuários médicos, antecipadamente acordados com os pacientes através da assinatura do Termo de Consentimento Livre e Esclarecido (TCLE).

\section{AMOSTRA}

Para a realização deste estudo, foi feito uma análise retrospectiva de um banco de dados de pacientes atendidos em um programa de reabilitação cardiopulmonar e metabólica (RCPM) do espaço Viver Bem (EVB) da Unimed - Juiz de Fora, MG. Para isso, foi analisado os parâmetros metabólicos de colesterol total, HDL, LDL e triglicérides de 105 indivíduos de ambos os sexos (39 mulheres com idade média de $67,3 \pm 8,0$ anos e 66 homens com idade média de 68,1 $\pm 8,4$ anos) nos prontuários clínicos dos pacientes atendidos durante todo o ano de 2016. Como critérios de inclusão para realização deste estudo foram considerados os respectivos fatores: a) todos os pacientes deveriam ser diagnosticados com dislipidemia, incluídos na RCPM fase III por indicação de seu médico assistente; b) Possuir todas as variáveis metabólicas analisadas nos períodos de pré e pós-intervenção registradas em seus prontuários; c) Possuir frequência de pelo menos $75 \%$ nas sessões de trei- 
namento físico e ter frequentando o programa de RCPM por 10 meses ininterruptos.

Foi considerada como dislipidemia a alteração de pelo menos um dos seguintes parâmetros bioquímicos: colesterol total (CT) com valores acima de $200 \mathrm{mg} / \mathrm{dl}$, triglicérides (TG) com valores acima de $150 \mathrm{mg} / \mathrm{dl}$, LDL-colesterol (LDL-C) com valores acima de $100 \mathrm{mg} / \mathrm{dl}$, HDL-colesterol (HDL-C) com valores abaixo de $40 \mathrm{mg} / \mathrm{dl}$ para homens e $50 \mathrm{mg} / \mathrm{dl}$ para mulheres ${ }^{2}$.

Todos os dados utilizados neste estudo foram autorizados pela diretoria da empresa privada de saúde complementar.

\section{PROCEDIMENTOS DA REABILITAÇÃO CARDIOPULMONAR E METABÓLICA}

Após a anamnese inicial que consta da verificação dos exames bioquímicos os pacientes são direcionados para a fase de reabilitação mais apropriada, no caso da presente intervenção é a fase III.

A RCPM fase III é um programa de exercício físico supervisionado por profissionais de Educação Física que direcionam a prescrição das sessões de treinamento. Especificamente, as sessões de treinamento são constituídas na sequência de exercícios aeróbicos, resistidos e de alongamento duas vezes por semana com 1 hora de duração. Para a sessão de treinamento de força (TF) foram utilizados exercícios de grandes grupamentos progredindo em direção aos pequenos grupamentos musculares compostos por até oito exercícios, tais como, exercícios de agachamento, remadas, flexão de joelho, elevação lateral, exercícios abdominais e de flexão plantar. Todas as sessões consistiam dos indivíduos realizarem três séries entre 10 e 12 repetições submáximas (de acordo com de percepção de esforço de cada paciente pela escala de OMNI-RES com pontuação entre 7 e 8) e intervalo de 1 minuto entre as séries e exercícios. Já as sessões de treinamento aeróbico foram realizadas durante 30 minutos parcelados (15 minutos de esteira e 15 minutos de cicloergômetro) com intensidade entre 60 e $80 \%$ da frequência cardíaca de reserva controlada por cardiofrequencímetro (FS2, Polar ${ }^{\oplus}$, Finlândia).

\section{ANÁLISE ESTATÍSTICA}

Foi feito análise de normalidade pelo teste Shapiro wilk para verificar a homogeneidade dos dados (critério de Bartlet). Foi utilizado o test $t$ pareado para analisar as diferenças nas medidas dos períodos de pré e pós-intervenção. Todos os dados são apresentados pela média e desvio-padrão. O nível de significância adotado foi o de $\mathrm{p} \leq 0,05$ através do software estatístico SPSS 20.0.

\section{RESULTADOS}

A tabela 1 apresenta os resultados das variáveis metabólicas nos períodos pre e pós-treinamento. Os resultados demonstram que após um período de 10 meses de treinamento os níveis de colesterol total, HDL, LDL e triglicérides melhoram significativamente $(\mathrm{p} \leq 0,05)$.

Tabela 1. Variáveis metabólicas no pré e pós-treinamento.

\begin{tabular}{llll}
\hline Variáveis Fisiológicas & Pré-treinamento & Pós-treinamento & Valor de p \\
\hline Colesterol total $(\mathrm{mg} / \mathrm{dl})$ & $185,0 \pm 45,9$ & $161,5 \pm 33,2^{*}$ & 0,001 \\
\hline HDL $(\mathrm{mg} / \mathrm{dl})$ & $43,2 \pm 11,2$ & $46,7 \pm 12,1^{\star}$ & 0,001 \\
\hline LDL $(\mathrm{mg} / \mathrm{dl})$ & $109,9 \pm 35,7$ & $92,4 \pm 26,5^{*}$ & 0,001 \\
\hline Triglicérides $(\mathrm{mg} / \mathrm{dl})$ & $149,0 \pm 63,7$ & $124,9 \pm 53,5^{*}$ & 0,001 \\
\hline *Indica diferença significativa em relação às medidas do pré-treinamento.
\end{tabular}

\section{DISCUSSÃO}

As pesquisas com intervenção através do exercício físico tem sido frequentemente o foco da maioria das investigações cientificas até mesmo por ser considerada uma terapia menos onerosa para o combate de diversas doenças. Entretanto, mesmo existindo muitas pesquisas na atualidade, os protocolos de treinamento ainda não são claros para a maioria das patologias. 
Por esta razão foi objetivo do presente estudo analisar os efeitos de um programa de exercícios físicos no perfil lipídico de indivíduos dislipidêmicos de ambos os sexos participantes de um programa multidisciplinar de reabilitação cardiopulmonar e metabólica. Os resultados do presente estudo demonstraram que após um período de 10 meses de RCPM é possível verificar diminuição nos níveis de colesterol total, LDL, triglicérides e aumento nos valores de HDL (tabela 1). Estas respostas são de grande importância, pois, qualquer alteração em um ou mais fatores no metabolismo dos lipídios podem contribuir para o desencadeamento de doenças cardíacas. Por exemplo, é bem definido na literatura a íntima relação entre LDL e manifestação de doenças ateroscleróticas ${ }^{9,10}$. Os mecanismos pelos quais a LDL participa deste processo podem estar relacionados a defeitos nos receptores desta lipoproteína o que resulta em uma não captação celular eficiente e consequente aumento da concentração plasmática da mesma ${ }^{9}$. Sendo assim, essa maior permanência da LDL no espaço subendotelial pode elevar a possibilidade de sofrer transformação em sua composição lipídica com potencial para iniciar o processo aterogênico ${ }^{11}$. Em contra partida, a prática de exercícios físicos pode contribuir para a oxidação da $\mathrm{LDL}^{12}$. O exercício físico atua no aumento do consumo de oxigênio o que pode favorecer para o crescimento do processo de oxidação dos valores de LDL, além de melhorar a complacência arterial, níveis de oxido nítrico, e fluxo sanguíneo ${ }^{12-14}$. Estas respostas fisiológicas positivas podem expor ao menor risco de desenvolvimento de doenças ateroscleróticas e consequente manutenção e/ou prevenção da saúde vascular.

Com relação ao tipo de exercício físico a grande maioria dos estudos realizados concentra-se nos exercícios de característica aeróbica ${ }^{15}$. Nesse sentido, o exercício aeróbico tem sido aplicado como de grande importância no tratamento não medicamentoso de indivíduos dislipidêmicos ${ }^{15,16}$. Contudo, existem poucas evidências científicas relacionando o TF sobre as respostas lipídicas ${ }^{17}$. Pois, as respostas verificadas na literatura são de redução do colesterol total e LDL ou manutenção dos valores de triglicérides ${ }^{17-20}$. Desta forma, o efeito do TF sobre a dislipidemia precisa ser mais explorado na literatura. No presente estudo, as sessões de treinamento também envolveram o TF e os resultados apresentados foram satisfatórios. Entretanto, o protocolo de treinamento foi realizado por uma combinação entre exercícios aeróbicos e de força o que não nos permitem distinguir um que seja mais efetivo. Independentemente disso, a prática regular de exercício físico pode proporcionar efeitos benéficos tais como diminuição nas concentrações plasmáticas de triglicérides, colesterol, LDL e aumento na concentração plasmática de HDL o que é de extrema relevância para a saúde vascular ${ }^{16,17}$.

Vale ressaltar que o presente estudo apresenta algumas limitações, por exemplo, o fato de se tratar de um estudo retrospectivo analítico observacional, não tem como garantir precisamente a maneira como foi executada a intervenção, assim como, as terapias farmacológicas e não farmacológicas utilizadas paralelas à RCPM.

De forma geral, os parâmetros hemodinâmicos avaliados no presente estudo mostraram que a prática regular de exercício físico, orientada por profissional de Educação Física em programas de RCPM, torna-se uma importante ferramenta na obtenção de benefícios relacionados ao metabolismo dos lipídeos para pacientes com dislipidemia.

\section{CONCLUSÃO}

Concluiu-se que, a adesão em um programa de RCPM para portadores de dislipidemia, por aproximadamente 10 meses, praticado duas vezes na semana, mostrou melhora no perfil de colesterol total, HDL, LDL e triglicérides. Porém, a realização de novos estudos randomizados e controlados é de fundamental importância para o entendimento dos mecanismos que envolvem o metabolismo dos lipídeos associados a um programa de treinamento físico.

\section{REFERÊNCIAS}

1. Organização Pan-americana da Saúde (OPAS). Doenças cardiovasculares. Revisado em setembro de 2016. Acesso em: http://www.paho.org/bra/index.php?option=com content\&view $=$ article\&id $=5253$ : doencas-cardiovascula res- $\&$ catid $=845:$ noticias $\&$ Itemid $=839$ 
2. V Diretrizes Brasileiras sobre Dislipidemias e Diretriz de Prevenção da Aterosclerose do Departamento de Aterosclerose da Sociedade Brasileira de Cardiologia. Arq Bras Cardiol. 2013; 77:1-48.

3. Giannini SD. Aterosclerose/Dislipidemias, Clínica e Terapêutica: Fundamentos Práticos. São Paulo: BG Cultural; 1998.

4. Prado ES, Dantas EHM. Efeitos dos exercícios físicos aeróbios e de força nas lipoproteínas HDL, LDL e lipoproteína (a). Arq Bras Cardiol. 2002; 79(4): 429-433.

5. American Heart Association (AHA). Resistance Exercise in Individuals With and Without Cardiovascular Disease: 2007 Update A Scientific Statement From the American Heart Association Council on Clinical Cardiology and Council on Nutrition, Physical Activity, and Metabolism. Circulation. 2007; 116(5):572-84.

6. American College of Sports Medicine (ACSM): position stand. Exercise and hypertension. Med Sci Sports Exerc. 2004;36:533-43.

7. Cardozo DC, de Souza Destro D, Cardozo LC. Influência do exercício físico no controle barorreflexo na hipertensão arterial. Rev Bras Presc Fisiol Exerc. 2012; 6(34): 342-349.

8. Cardozo D, Alves HB, Figueiredo T, Dias MR, Simão, R. Efeito hipotensivo no treinamento resistido: influência da massa muscular envolvida. ConScientiae Saúde. 2014; 13(4), 524-532.

9. Brown MS, Goldstein JL. (1986). A receptor-mediated pathway for cholesterol homeostasis. Science. 1986; 232(4746): 34-47.

10. Castelli WP, Garrison RJ, Wilson PW, Abbott RD, Kalousdian S, Kannel WB. Incidence of coronary heart disease and lipoprotein cholesterol levels: the Framingham Study. Jama. 1986; 256(20): 2835-2838.

11. Berliner JA, Navab M, Fogelman AM, Frank JS, Demer LL, Edwards PA, Lusis AJ. Atherosclerosis: Basic Mechanisms: Oxidation, Inflammation, and Genetics. ACC Current Journal Review. 1996; 3(5): 37.

12. Liu ML, Bergholm R, Mäkimattila S, Lahdenperä S, Valkonen M, Hilden H, Taskinen, M R. A marathon run increases the susceptibility of LDL to oxidation in vitro and modifies plasma antioxidants. Am J Physiol. 1999; 276(6): E1083-E1091.

13. Bai H, Sun J, Du G, Jiao F. Association of moderate aerobic exercise and rho-associated kinase 2 concentration in subjects with dyslipidemia. Arch Med Sci. 2017; 13(4): 807-812.
14. De Oliveira Sarmento A, da Cruz Santos A, Trombetta IC, Dantas MM, Marques ACO, do Nascimento LS, do Socorro Brasileiro-Santos M. Regular physical exercise improves cardiac autonomic and muscle vasodilatory responses to isometric exercise in healthy elderly. Clin Interv Aging. 2017;12, 1021-1028.

15. Goodyear LJ, Van Houten DR, Fronsoe MS, Rocchio ML, Dover EV, Durstine JL. Immediate and delayed effects of marathon running on lipids and lipoproteins in women. Med Sci Sports Exerc. 1990; 22(5): 588-592.

16. Berg A, Frey I, Baumstark MW, Halle M, Keul J. Physical activity and lipoprotein lipid disorders. Sports Med. 1994; 17(1): 6-21.

17. Bezerra A, Kanegusuku H, Prado W, Ritti-Dias R, Júnior CC. Efeito do exercício físico aeróbico e de força no perfil lipídico de seus praticantes: uma revisão sistemática. Rev Bras Ativ Fís e Saúde. 2013; 18(4): 399-400.

18. Boyden TW, Pamenter RW, Going SB, Lohman TG, Hall MC, Houtkooper LB, Aickin M. Resistance exercise training is associated with decreases in serum low-density lipoprotein cholesterol levels in premenopausal women. Arch Intern Med. 1993; 153(1): 97-100.

19. Gavin C, Sigal RJ, Cousins M, Menard ML, Atkinson M, Khandwala F, Ooi TC. Resistance exercise but not aerobic exercise lowers remnant-like lipoprotein particle cholesterol in type 2 diabetes: a randomized controlled trial. Atherosclerosis. 2010; 213(2): 552-557.

20. Polito MD, Cyrino ES, Gerage AM, Nascimento MAD, Januário RSB. 12-week resistance training effect on muscular strength, body composition and triglycerides in sedentary men. Rev Bras Med Esporte. 2010; 16(1): 29-32. 